\title{
Stability and Boundedness of Stochastic Volterra Integrodifferential Equations with Infinite Delay
}

\author{
Chunmei Zhang, Wenxue Li, and Ke Wang \\ Department of Mathematics, Harbin Institute of Technology (Weihai), Weihai 264209, China \\ Correspondence should be addressed to Wenxue Li; wenxuetg@hitwh.edu.cn
}

Received 28 November 2012; Accepted 13 February 2013

Academic Editor: Jinde Cao

Copyright (c) 2013 Chunmei Zhang et al. This is an open access article distributed under the Creative Commons Attribution License, which permits unrestricted use, distribution, and reproduction in any medium, provided the original work is properly cited.

\begin{abstract}
We make the first attempt to discuss stability and boundedness of solutions to stochastic Volterra integrodifferential equations with infinite delay (IDSVIDEs). By the Lyapunov-Krasovskii functional approach, we get kinds of sufficient criteria for stability and boundedness of solutions to IDSVIDEs. The main innovation here is that stochastic systems with infinite delay can retain stability and boundedness of corresponding deterministic systems under some conditions.
\end{abstract}

\section{Introduction}

Recently, stochastic functional differential equations with infinite delay (IDSFDEs) have attracted broad attention of many researchers. In the literature, there are two main lines of research on the IDSFDEs. On one hand, existence and uniqueness of solution are basic properties for equations. So a great number of authors have devoted themselves to this research, and thus many excellent results on the existence and uniqueness of the solutions to IDSFDEs and neutral IDSFDEs can be found in [1-6] and references cited therein. On the other hand, the study of stability and boundedness of solutions is one of the most attracting topics in the qualitative theory of differential equations because of its various applications in many areas such as physics and control theory $[7,8]$. Hence, more and more researchers study them and especially focus on the stability of solutions. An important issue in stochastic analysis is whether or not random disturbance can change the qualitative properties of system, which is particularly important in control field. In most cases, people are interested in the performance of antidisturbance of system. So it is vital to seek some antidisturbance systems or present the intensity of stochastic perturbation that stable system can tolerate without losing the property of stability [9]. In recent years, many meaningful works on this topic have come out; see, for example, [1021].
Volterra integrodifferential equations (VIDEs) are widely applied in biology, ecology, medicine, physics, among other scientific areas and thus have been encountered by many researchers in numerical and theoretic analysis; see [7, 2225]. It is well known that concrete systems are inevitably affected by external perturbations usually modeled by stochastic noise. So a great deal of attention has been paid to the research of stochastic VIDEs [9, 26, 27]. Additionally, time delay is always ubiquitous and infinite delay systems have wide applications in many fields. Hence, there is naturally an important kind of IDSFDEs, that is, stochastic Volterra integrodifferential equations with infinite delay (IDSVIDEs). In practice, many applications of IDSVIDEs are greatly dependent on the stability and boundedness of their solutions. However, to the best of the authors' knowledge, few research results mentioned above focus on the stability and boundedness of IDSVIDEs, which motivates the present study. Precisely, this paper investigates in detail the problem of stability and boundedness of solutions for the following IDSVIDE:

$$
\begin{aligned}
\mathrm{d} x(t)= & {\left[A(t) x(t)+\int_{-\infty}^{t} C(t, s) g(x(s)) \mathrm{d} s+f(t)\right] \mathrm{d} t } \\
& +[B(t) x(t) \\
& \left.\quad+\int_{-\infty}^{t} D(t, s) g(x(s)) \mathrm{d} s+f_{1}(t)\right] \mathrm{d} W(t), \quad t \geq t_{0},
\end{aligned}
$$


where $A(t), B(t), f(t)$, and $f_{1}(t): \mathbb{R} \rightarrow \mathbb{R}$ are continuous functions. $C(t, s)$ and $D(t, s): \mathbb{R} \times \mathbb{R} \rightarrow \mathbb{R}$ are also continuous. Therein, $B(t), D(t, s)$, and $f_{1}(t)$ denote the intensity of disturbance to $A(t), C(t, s)$, and $f(t)$, respectively.

Compared with the existing results in the literature, contributions of this paper are mainly as follows.

(1) Both stochastic perturbation and infinite delay are considered in the IDSVIDEs.

(2) A new Lyapunov function is constructed to derive stability and boundedness criteria for IDSVIDEs efficiently.

(3) The problem of how much the stochastic noise VIDEs with infinite delay can tolerate without losing the properties of stability and boundedness has been solved.

\section{Preliminaries}

Let $(\Omega, \mathscr{F}, \mathbb{F}, \mathbb{P})$ be a complete probability space with a filtration $\mathbb{F}=\left\{\mathscr{F}_{t}\right\}_{t \geq 0}$ satisfying the usual conditions. As usual, $W(\cdot)$ denotes a scalar Brownian motion defined on the space and $\mathbb{E}(\cdot)$ is the mathematical expectation with respect to $\mathbb{P}$. Write $B C((-\infty, 0] ; \mathbb{R})$ as the family of bounded continuous real-valued functions $\phi$ defined on $(-\infty, 0]$ with the norm $\|\phi\|=\sup _{-\infty<\theta \leq 0}|\phi(\theta)|$.

In this paper, we suppose there exists a constant $J>0$ such that $|g(x)| \leq J|x|^{2}$. Then by Theorem 5.2.7 of [28], there exists a unique global solution $x(t)$ to $(1)$ if $A(t)$ and $B(t)$ are bounded. For more details, readers can see $[29,30]$. Here and in the rest of the paper, write the solutions with the initial condition $x_{t_{0}}=\phi \in B C((-\infty, 0] ; \mathbb{R})$ as $x\left(t ; t_{0}, \phi\right)$. Throughout this paper, unless otherwise specified, we use the following Lyapunov function:

$$
V(x, t)=\frac{x^{2}}{2}+k \int_{-\infty}^{t} \int_{t}^{+\infty}|C(u, s)||g(x(s))| \mathrm{d} u \mathrm{~d} s .
$$

For any $M>0$, define two stopping times:

$$
\begin{aligned}
& \tau_{M}=\inf \left\{t \geq t_{0}:|x(t)| \leq M\right\}, \\
& \tau^{M}=\inf \left\{t \geq t_{0}:|x(t)| \geq M\right\} .
\end{aligned}
$$

In order to study the stability and boundedness of solutions to (1), we state the following assumptions:

(K1) There exist nonnegative continuous functions $h$ : $\mathbb{R}^{-} \rightarrow \mathbb{R}^{+}$and $F: \mathbb{R} \rightarrow \mathbb{R}$, such that

$$
\begin{aligned}
& \int_{-\infty}^{0} h(s) \mathrm{d} s=l<\infty, \quad|C(t, s)| \leq F(t) h(s-t), \\
& \int_{t}^{+\infty} F(u) \mathrm{d} u \leq a<\infty, \quad \lim _{t \rightarrow \infty} \int_{t}^{+\infty} F(u) \mathrm{d} u=0 .
\end{aligned}
$$

(K2) For any $\alpha \in(0,1)$, there exists $k>1$ such that

$$
A(t)+B^{2}(t)+k J \int_{t}^{+\infty}|C(u, t)| \mathrm{d} u \leq 0
$$

$$
m(t) D(t, s)<\frac{k-\alpha}{\sup _{|x| \leq \alpha}|g(x)|}|C(t, s)|,
$$

where $m(t):=\int_{-\infty}^{t}|D(t, s)| \mathrm{d} s$.

(K3) For any $\alpha \in(0,1)$, there exist $k>1$ and $\delta>0$ such that

$$
\begin{aligned}
& A(t)+B^{2}(t)+k J \int_{t}^{+\infty}|C(u, t)| \mathrm{d} u<-\delta, \\
& m(t) D(t, s)<\frac{k-\alpha}{\sup _{|x| \leq \alpha}|g(x)|}|C(t, s)|,
\end{aligned}
$$

where $m(t)$ is defined in (K2).

Remark 1. Conditions (K2) and (K3) are the conditions about the intensity of perturbations. Note that constant $k$ in equality (2) is the same as the one which appeared in conditions (K2) and (K3).

\section{Stability of Solutions of IDSVIDEs}

In order to consider the stability of (1), without loss of generality, we suppose that $f(t)=f_{1}(t)=0$ and $g(0)=0$ hold in this section. Hence, (1) has the trivial solution $x(t) \equiv$ 0 . First, we introduce three kinds of definitions about stability of solutions to (1). It is easy to see that these definitions are a strict generalization of deterministic cases.

Definition 2 (see [28]). (1) The trivial solution of (1) is said to be stochastically stable if for every pair $\varepsilon \in(0,1)$ and $r>0$, there exists a $\delta=\delta\left(\varepsilon, r, t_{0}\right)>0$ such that

$$
\mathbb{P}\left(\left|x\left(t ; t_{0}, \phi\right)\right|<r, t \geq t_{0}\right) \geq 1-\varepsilon
$$

whenever $\|\phi\|<\delta$. Additionally, it is said to be stochastically uniformly stable if $\delta$ is independent of $t_{0}$.

(2) The trivial solution of (1) is said to be stochastically asymptotically stable if it is stochastically stable and, moreover, for any $\varepsilon \in(0,1)$, there is $\delta_{0}=\delta_{0}\left(\varepsilon, t_{0}\right)>0$ such that

$$
\mathbb{P}\left(\lim _{t \rightarrow \infty} x\left(t ; t_{0}, \phi\right)=0\right) \geq 1-\varepsilon,
$$

whenever $\|\phi\|<\delta_{0}$.

(3) The trivial solution of (1) is said to be stochastically globally asymptotically stable if it is stochastically stable and, moreover, for all $\phi \in B C((-\infty, 0] ; \mathbb{R})$, it follows that

$$
\mathbb{P}\left(\lim _{t \rightarrow \infty} x\left(t ; t_{0}, \phi\right)=0\right)=1 \text {. }
$$

In the following, we will apply the Lyapunov-Krasovskii functional approach to delve into some sufficient criteria, under which the trivial solution to (1) is stochastically stable, stochastically asymptotically stable, and stochastically globally asymptotically stable, respectively. The Itô formula used in this paper can be seen in [28].

Theorem 3. Suppose that (K1) and (K2) hold. Then the trivial solution of (1) is stochastically uniformly stable. 
Proof. For any $\varepsilon \in(0,1)$ and $r \in(0,1)$, choose $\delta$ sufficiently small for $\delta<r^{2} \varepsilon /(1+2 k J l a)$. For any given $\phi \in B C((-\infty, 0] ; \mathbb{R})$ satisfying $\|\phi\| \leq \delta$, we can get from (2) that

$$
\begin{aligned}
\mathscr{L} V & (x(s), s) \\
= & V_{t}(x(s), s)+V_{x}(x(s), s) \\
& \times\left[A(s) x(s)+\int_{-\infty}^{s} C(s, u) g(x(u)) \mathrm{d} u\right] \\
& +\frac{1}{2} V_{x x}(x(s), s)\left[B(s) x(s)+\int_{-\infty}^{s} D(s, u) g(x(u)) \mathrm{d} u\right]^{2} \\
= & k \int_{s}^{+\infty}|C(u, s)||g(x(s))| \mathrm{d} u \\
& -k \int_{-\infty}^{s}|C(s, u)||g(x(u))| \mathrm{d} u+A(s) x^{2}(s) \\
& +x(s) \int_{-\infty}^{s} C(s, u) g(x(u)) \mathrm{d} u \\
& +\frac{1}{2}\left[B(s) x(s)+\int_{-\infty}^{s} D(s, u) g(x(u)) \mathrm{d} u\right]^{2} \\
\leq & \left(k J \int_{s}^{+\infty}|C(u, s)| \mathrm{d} u+A(s)+B^{2}(s)\right) x^{2}(s) \\
& -(k-|x(s)|) \int_{-\infty}^{s}|C(s, u)||g(x(u))| \mathrm{d} u \\
& +\left[\int_{-\infty}^{s} D(s, u) g(x(u)) \mathrm{d} u\right]^{2} .
\end{aligned}
$$

Then it follows that, for any $t \geq t_{0}$,

$$
\begin{aligned}
& \mathbb{E} V\left(x\left(t \wedge \tau^{r}\right), t \wedge \tau^{r}\right) \\
& =V\left(\phi\left(t_{0}\right), t_{0}\right) \\
& +\mathbb{E} \int_{t_{0}}^{t \wedge \tau^{r}}\left[\left(k J \int_{s}^{+\infty}|C(u, s)| \mathrm{d} u+A(s)+B^{2}(s)\right) x^{2}(s)\right. \\
& -(k-|x(s)|) \int_{-\infty}^{s}|C(s, u)||g(x(u))| \mathrm{d} u \\
& \left.+\left[\int_{-\infty}^{s} D(s, u) g(x(u)) d u\right]^{2}\right] \mathrm{d} s \\
& \leq V\left(\phi\left(t_{0}\right), t_{0}\right) \\
& +\mathbb{E} \int_{t_{0}}^{t \wedge \tau^{r}}\left[\left(k J \int_{s}^{+\infty}|C(u, s)| \mathrm{d} u+A(s)+B^{2}(s)\right) x^{2}(s)\right.
\end{aligned}
$$

$$
\begin{aligned}
& +\int_{-\infty}^{s}[-(k-r)|C(s, u)| \\
& \left.+\left(\sup _{|x| \leq r}|g(x)|\right) m(s)|D(s, u)|\right] \\
& \times|g(x(u))| \mathrm{d} u] \mathrm{d} s \\
& \leq V\left(\phi\left(t_{0}\right), t_{0}\right) .
\end{aligned}
$$

Then by a straightforward computation we obtain that

$$
\begin{aligned}
V\left(\phi\left(t_{0}\right), t_{0}\right) & =\frac{\phi^{2}\left(t_{0}\right)}{2}+k \int_{-\infty}^{t_{0}} \int_{t_{0}}^{+\infty}|C(u, s)||g(x(s))| \mathrm{d} u \mathrm{~d} s \\
& \leq\left(\frac{1}{2}+k J l a\right)\|\phi\|^{2}<\frac{r^{2}}{2} \varepsilon .
\end{aligned}
$$

From the definition of $\tau^{r}$, it follows that

$$
\begin{aligned}
\mathbb{E} V\left(x\left(t \wedge \tau^{r}\right), t \wedge \tau^{r}\right) & \geq \mathbb{E}\left(I_{\left\{\tau^{r}<t\right\}} V\left(x\left(\tau^{r}\right), \tau^{r}\right)\right) \\
& \geq \frac{r^{2}}{2} \mathbb{P}\left(\tau^{r}<t\right) .
\end{aligned}
$$

So

$$
\mathbb{P}\left(\tau^{r}<t\right)<\varepsilon .
$$

Let $t \rightarrow \infty$. We deduce that

$$
\mathbb{P}\left(\tau^{r}<\infty\right) \leq \varepsilon,
$$

that is,

$$
\mathbb{P}\left(\left|x\left(t ; t_{0}, \phi\right)\right| \leq r, t \geq t_{0}\right)>1-\varepsilon .
$$

For any $r_{1} \in[1,+\infty)$, we can have

$$
\left\{\omega|| x\left(t ; t_{0}, \phi\right) \mid \leq r, t \geq t_{0}\right\} \subseteq\left\{\omega|| x\left(t ; t_{0}, \phi\right) \mid \leq r_{1}, t \geq t_{0}\right\},
$$

which implies that

$$
\begin{aligned}
\mathbb{P}\left(\left|x\left(t ; t_{0}, \phi\right)\right| \leq r_{1}, t \geq t_{0}\right) & \geq \mathbb{P}\left(\left|x\left(t ; t_{0}, \phi\right)\right| \leq r, t \geq t_{0}\right) \\
& >1-\varepsilon .
\end{aligned}
$$

This completes the proof.

Remark 4. If $B(t)=D(t, s)=0$ in (1), then we can get the corresponding disturbance free system. Theorem 3 really tells us that stochastic perturbation cannot disturb the stability of original deterministic system if the noisy intensity satisfies (K2).

Lemma 5. Assume that (K1) and (K2) hold. Then for any $\varepsilon \in$ $(0,1), \alpha>0$, there is $R(\varepsilon, \alpha)>0$ such that

$$
\mathbb{P}\left(\left|x\left(t ; \theta^{*}, \phi\right)\right| \leq R, t \geq \theta^{*}\right)>1-\varepsilon,
$$

for any $\theta^{*} \geq t_{0},\|\phi\|<\alpha$, a.s. 
Proof. For any given $\varepsilon \in(0,1), \alpha>0$, and $\phi \epsilon$ $B C((-\infty, 0] ; \mathbb{R})$ satisfying $\|\phi\| \leq \alpha$, choose $R(\varepsilon, \alpha)$ sufficiently large such that

$$
R^{2}>\frac{\alpha^{2}(1+2 k J l a)}{\varepsilon} .
$$

Denote $x(t) \triangleq x\left(t ; \theta^{*}, \phi\right)$. By using a similar argument as in Theorem 3, we have that, for any $t \geq \theta^{*}$,

$$
\begin{aligned}
\mathbb{E} V(x & \left.\left(t \wedge \tau^{R}\right), t \wedge \tau^{R}\right) \\
& \leq \mathbb{E} V\left(\phi\left(\theta^{*}\right), \theta^{*}\right) \\
& =\frac{\mathbb{E} \phi^{2}\left(\theta^{*}\right)}{2}+k \mathbb{E} \int_{-\infty}^{\theta^{*}} \int_{\theta^{*}}^{+\infty}|C(u, s)||g(x(s))| \mathrm{d} u \mathrm{~d} s \\
& \leq \frac{\mathbb{E} \phi^{2}\left(\theta^{*}\right)}{2}+k J l a \mathbb{E}\|\phi\|^{2} \\
& \leq\left(\frac{1}{2}+k J l a\right) \mathbb{E}\|\phi\|^{2} .
\end{aligned}
$$

Making use of the definition of $V(x, t)$ yields

$$
\begin{aligned}
\mathbb{E} V\left(x\left(t \wedge \tau^{R}\right), t \wedge \tau^{R}\right) & \geq \mathbb{E}\left(I_{\left\{\tau^{R}<t\right\}} V\left(x\left(\tau^{R}\right), \tau^{R}\right)\right) \\
& \geq \frac{R^{2}}{2} \mathbb{P}\left(\tau^{R}<t\right) .
\end{aligned}
$$

Combining this and (21), we have

$$
\mathbb{P}\left(\tau^{R}<t\right) \leq \frac{(1+2 k J l a) \mathbb{E}\|\phi\|^{2}}{R^{2}}<\varepsilon .
$$

Let $t \rightarrow \infty$. Then

$$
\mathbb{P}\left(\tau^{R}<\infty\right) \leq \varepsilon,
$$

which can imply our desired result

$$
\mathbb{P}\left(\left|x\left(t ; \theta^{*}, \phi\right)\right| \leq R, t \geq \theta^{*}\right)>1-\varepsilon .
$$

Theorem 6. Suppose that (K1) and (K3) hold. Then the trivial solution is stochastically asymptotically stable and stochastically globally asymptotically stable.

Proof. (I) The proof of stochastic asymptotic stability.

For any $\theta^{*}>t_{0}, \phi^{*}=\left\{\phi^{*}(\theta):-\infty<\theta \leq 0\right\}$ is $\mathscr{F}_{\theta^{*}}$-adapted $B C((-\infty, 0] ; \mathbb{R})$-valued random variable such that $\mathbb{E}\left\|\phi^{*}(\theta)\right\|<\infty$. Let $x\left(t ; \theta^{*}, \phi^{*}\right)$ denote the solution with the initial value $\left(\theta^{*}, \phi^{*}\right)$. From Theorem 3 , the trivial solution is stochastically uniformly stable. Hence, for any given $\delta_{1}>$ $0, \varepsilon \in(0,1)$, there exists $\delta\left(\varepsilon, \delta_{1}\right)>0$, such that

$$
\mathbb{P}\left(\omega:\left|x\left(t ; t_{0}, \phi\right)\right|<\delta_{1}, t \geq t_{0}\right) \triangleq \mathbb{P}(A)>1-\varepsilon,
$$

whenever $\|\phi\|<\delta\left(\varepsilon, \delta_{1}\right)$. Fix $\phi \in B C((-\infty, 0] ; \mathbb{R})$ such that $\|\phi\|<\delta\left(\varepsilon, \delta_{1}\right)$. For any given $\omega^{*} \in A, \sigma \geq t_{0}$, define

$$
\begin{aligned}
\phi_{\sigma}(t, \omega)= & x\left(t ; t_{0}, \phi\right) I_{(t, \omega) \in(-\infty, \sigma] \times A} \\
& +x\left(t ; t_{0}, \phi, \omega^{*}\right) I_{(t, \omega) \in(-\infty, \sigma] \times(\Omega-A)} .
\end{aligned}
$$

So Lemma 5 shows that, for $\delta_{1}$ above and any $\varepsilon_{1} \in(0,1)$, there is $H\left(\varepsilon_{1}, \delta_{1}\right)$ such that

$$
\mathbb{P}\left(\left|x\left(t ; \sigma, \phi_{\sigma}\right)\right| \leq H, t \geq \sigma\right)>1-\frac{\varepsilon_{1}}{4} .
$$

If there exists $K>t_{0}$ such that

$$
\mathbb{P}\left(\omega \in A:\left|x\left(t ; t_{0}, \phi\right)\right|=0, t \geq K\right)=\mathbb{P}(A)>1-\varepsilon,
$$

then the trivial solution of system (1) is stochastically asymptotically stable. If not, there are a sequence $\gamma_{k}$ and increasing sequence $\tau_{k}$ such that $\lim _{k \rightarrow \infty} \tau_{k}=+\infty$ and

$$
\mathbb{P}\left(\omega \in A:\left|x\left(\tau_{k} ; t_{0}, \phi\right)\right| \neq 0\right)=\gamma_{k}>0, \quad k=1,2, \ldots
$$

Define

$$
S_{k}^{\beta}=\left(\omega \in A:\left|x\left(\tau_{k} ; t_{0}, \phi\right)\right|>\beta\right), \quad k=1,2, \ldots
$$

It is not difficult to see that

$$
\lim _{\beta \rightarrow 0} \mathbb{P}\left(S_{k}^{\beta}\right)=\gamma_{k}, \quad k=1,2, \ldots
$$

Hence, there are $\beta_{0}>0$ and positive sequence $\gamma_{k}^{0}$ such that

$$
\begin{aligned}
& \mathbb{P}\left(\omega \in A:\left|x\left(\tau_{k} ; t_{0}, \phi\right)\right|>\beta_{0}\right) \\
& \quad \triangleq \mathbb{P}\left(A_{\tau_{k}}\right)=\gamma_{k}^{0}>0, \quad k=1,2, \ldots
\end{aligned}
$$

If for any $\beta_{1} \in\left(0, \beta_{0}\right)$, the following holds:

$$
\mathbb{P}\left(\omega \in A: \limsup _{t \rightarrow \infty}\left|x\left(t ; t_{0}, \phi\right)\right|<\beta_{1}\right)>1-\varepsilon,
$$

which can show the trivial solution of system (1) is stochastically asymptotically stable. If not, there is $\beta_{2} \in\left(0, \beta_{0}\right)$ such that

$$
\mathbb{P}\left(\omega \in A: \limsup _{t \rightarrow \infty}\left|x\left(t ; t_{0}, \phi\right)\right|<\beta_{2}\right) \triangleq \mathbb{P}\left(B_{\beta_{2}}\right) \leq 1-\varepsilon .
$$

Obviously, $\mathbb{P}\left(A-B_{\beta_{2}}\right)>0, A_{\tau_{k}} \subseteq A-B_{\beta_{2}}$, and for any $\omega \epsilon$ $A-B_{\beta_{2}}$,

$$
\limsup _{t \rightarrow \infty}\left|x\left(t ; t_{0}, \phi\right)\right| \geq \beta_{2}>0 \text {. }
$$

For any given $B<\beta_{2}, \alpha<B$ such that $2 \alpha^{2} / B^{2}<\varepsilon_{1} / 4$. Choose $N$ sufficiently large such that, for any $t>\tau_{N}$,

$$
\int_{t}^{+\infty} F(u) d u<\frac{\alpha^{2}}{2 k l J H^{2}}
$$

For any given $\omega^{*} \in A_{\tau_{N}}$, define

$$
\begin{aligned}
\phi_{\tau_{N}}(t, \omega)= & x\left(t ; t_{0}, \phi\right) I_{(t, \omega) \in\left(-\infty, \tau_{N}\right] \times A_{\tau_{N}}} \\
& +x\left(t ; t_{0}, \phi, \omega^{*}\right) I_{(t, \omega) \in\left(-\infty, \tau_{N}\right] \times\left(\Omega-A_{\tau_{N}}\right)}
\end{aligned}
$$


So $(28)$ can tell us that

$$
\begin{gathered}
\mathbb{P}\left(\omega:\left|x\left(t ; \tau_{N}, \phi_{\tau_{N}}\right)\right|<H, t \geq \tau_{N}\right)>1-\frac{\varepsilon_{1}}{4}, \\
x\left(t ; \tau_{N}, \phi_{\tau_{N}}\right)=x\left(t ; t_{0}, \phi\right),(t, \omega) \in(-\infty,+\infty) \times A_{\tau_{N}} .
\end{gathered}
$$

From here, we let $x(t) \triangleq x\left(t ; \tau_{N}, \phi_{\tau_{N}}\right), t \geq \tau_{N}$, and let $V(x, t)$ be the same as (2).

By The Itô formula and (K3), for any $t \geq \tau_{N}$,

$$
\begin{aligned}
& \mathbb{E} V\left(x\left(t \wedge \tau_{\alpha} \wedge \tau^{H}\right), t \wedge \tau_{\alpha} \wedge \tau^{H}\right) \\
& \quad=V\left(\phi_{\tau_{N}}\left(\tau_{N}\right), \tau_{N}\right)+\mathbb{E} \int_{\tau_{N}}^{t \wedge \tau_{\alpha} \wedge \tau^{H}} \mathscr{L} V(x(s), s) \mathrm{d} s \\
& \quad \leq V\left(\phi_{\tau_{N}}\left(\tau_{N}\right), \tau_{N}\right)-\delta \alpha^{2} \mathbb{E}\left(t \wedge \tau_{\alpha} \wedge \tau^{H}-\tau_{N}\right)
\end{aligned}
$$

where $\mathscr{L} V(x(s), s)$ is the same as $(10)$. So,

$$
\begin{aligned}
\left(t-\tau_{N}\right) \mathbb{P}\left\{\tau_{\alpha} \wedge \tau^{H} \geq t\right\} & \leq \mathbb{E}\left(t \wedge \tau_{\alpha} \wedge \tau^{H}-\tau_{N}\right) \\
& \leq \frac{V\left(\phi_{\tau_{N}}\left(\tau_{N}\right), \tau_{N}\right)}{\delta \alpha^{2}} .
\end{aligned}
$$

Letting $t \rightarrow \infty$ can yield

$$
\mathbb{P}\left\{\tau_{\alpha} \wedge \tau^{H}<\infty\right\}=1
$$

Clearly, it follows from (39) that $\mathbb{P}\left(\tau^{H}<\infty\right) \leq \varepsilon_{1} / 4$. Therefore,

$$
\begin{aligned}
1= & \mathbb{P}\left(\tau_{\alpha} \wedge \tau^{H}<\infty\right) \leq \mathbb{P}\left(\tau_{\alpha}<\infty\right) \\
& +\mathbb{P}\left(\tau^{H}<\infty\right) \leq \mathbb{P}\left(\tau_{\alpha}<\infty\right)+\frac{\varepsilon_{1}}{4} .
\end{aligned}
$$

Hence,

$$
\mathbb{P}\left(\tau_{\alpha}<\infty\right) \geq 1-\frac{\varepsilon_{1}}{4} .
$$

Choose $\theta_{\alpha}$ sufficiently large such that

$$
\mathbb{P}\left(\tau_{\alpha}<\theta_{\alpha}\right) \geq 1-\frac{\varepsilon_{1}}{2} .
$$

Then

$$
\begin{aligned}
\mathbb{P}\left(\tau_{\alpha}<\tau^{H} \wedge \theta_{\alpha}\right) & \geq \mathbb{P}\left(\left\{\tau_{\alpha}<\theta_{\alpha}\right\} \cap\left\{\tau^{H}=\infty\right\}\right) \\
& \geq \mathbb{P}\left(\tau_{\alpha}<\theta_{\alpha}\right)-P\left(\tau^{H}<\infty\right) \geq 1-\frac{3 \varepsilon_{1}}{4} .
\end{aligned}
$$

Define two stopping times:

$$
\sigma=\left\{\begin{array}{ll}
\tau_{\alpha}, & \tau_{\alpha}<\tau^{H} \wedge \theta_{\alpha} ; \\
\infty, & \text { otherwise, }
\end{array} \quad \tau_{B}=\inf \{t>\sigma:|x(t)| \geq B\}\right.
$$

So for any $t \geq \theta_{\alpha}$,

$$
\mathbb{E} V\left(x\left(\tau_{B} \wedge t\right), \tau_{B} \wedge t\right) \leq \mathbb{E} V(x(\sigma \wedge t), \sigma \wedge t) .
$$

Note that if $\omega \in\left\{\tau_{\alpha} \geq \tau^{H} \wedge \theta_{\alpha}\right\}$, then

$$
V\left(x\left(\tau_{B} \wedge t\right), \tau_{B} \wedge t\right)=V(x(\sigma \wedge t), \sigma \wedge t) .
$$

Consequently,

$$
\begin{gathered}
\mathbb{E}\left(I_{\left\{\tau_{\alpha}<\tau^{H} \wedge \theta_{\alpha}\right\}} V\left(x\left(\tau_{B} \wedge t\right), \tau_{B} \wedge t\right)\right) \\
\leq \mathbb{E}\left(I_{\left\{\tau_{\alpha}<\tau^{H} \wedge \theta_{\alpha}\right\}} V\left(x\left(\tau_{\alpha}\right), \tau_{\alpha}\right)\right) .
\end{gathered}
$$

Noting $\left\{\tau_{B}<t\right\} \subseteq\left\{\tau_{\alpha}<\tau^{H} \wedge \theta_{\alpha}\right\}$, it yields that

$$
\begin{aligned}
& \mathbb{E}\left(I_{\left\{\tau_{\alpha}<\tau_{B} \wedge \theta_{\alpha}\right\}} V\left(x\left(\tau_{B} \wedge t\right), \tau_{B} \wedge t\right)\right) \\
& \quad \geq\left(\mathbb{E}\left(I_{\left\{\tau_{\alpha}<\tau_{B} \wedge \theta_{\alpha}\right\}} V\left(x\left(\tau_{B} \wedge t\right), \tau_{B} \wedge t\right)\right) \mid \tau_{B}<t\right) \mathbb{P}\left(\tau_{B}<t\right) \\
& \quad \geq \frac{B^{2}}{2} \mathbb{P}\left(\tau_{B}<t\right) .
\end{aligned}
$$

In view of the definition of $V(x, t)$ and condition (K1),

$$
\begin{aligned}
& \mathbb{E}\left(I_{\left\{\tau_{\alpha}<\tau^{H} \wedge \theta_{\alpha}\right\}} V\left(x\left(\tau_{\alpha}\right), \tau_{\alpha}\right)\right) \leq \mathbb{E} V\left(x\left(\tau_{\alpha}\right), \tau_{\alpha}\right) \\
& =\mathbb{E}\left[\frac{x^{2}\left(\tau_{\alpha}\right)}{2}+k \int_{-\infty}^{\tau_{\alpha}} \int_{\tau_{\alpha}}^{+\infty}|C(u, s)||g(x(s))| \mathrm{d} u \mathrm{~d} s\right] \\
& \leq \mathbb{E}\left[\frac{\alpha^{2}}{2}+k J H^{2} \int_{\tau_{\alpha}}^{+\infty} \int_{-\infty}^{\tau_{\alpha}} F(u) h(s-u) \mathrm{d} s \mathrm{~d} u\right] \\
& \leq \frac{\alpha^{2}}{2}+k J l H^{2} \int_{\tau_{\alpha}}^{+\infty} F(u) \mathrm{d} u \\
& <\alpha^{2},
\end{aligned}
$$

which, in conjunction with (51) and (52), yields that

$$
\mathbb{P}\left(\tau_{B}<t\right)<\frac{2 \alpha^{2}}{B^{2}}<\frac{\varepsilon_{1}}{4} .
$$

Letting $t \rightarrow \infty$, it yields that

$$
\mathbb{P}\left(\tau_{B}<\infty\right) \leq \frac{\varepsilon_{1}}{4} .
$$

Hence,

$$
\begin{aligned}
& \mathbb{P}\left(\{\sigma<\infty\} \cap\left\{\tau_{B}=\infty\right\}\right) \\
& \quad \geq \mathbb{P}\left(\tau_{\alpha}<\tau^{H} \wedge \theta\right)-\mathbb{P}\left(\tau_{B}<\infty\right) \geq 1-\varepsilon_{1} .
\end{aligned}
$$

This implies immediately that

$$
\mathbb{P}\left(\limsup _{t \rightarrow \infty}|x(t)| \leq B\right) \geq 1-\varepsilon_{1} .
$$

At last, from the arbitrariness of $B$, it must be

$$
\mathbb{P}\left(\limsup _{t \rightarrow \infty}|x(t)|=0\right) \geq 1-\varepsilon_{1} .
$$


Since $\varepsilon_{1}$ is arbitrary, we then obtain that

$$
\mathbb{P}\left(\limsup _{t \rightarrow \infty}|x(t)|=0\right)=1 .
$$

Hence, from (40), there is $\omega \in A_{\tau_{N}} \subseteq A-B_{\beta_{2}}$ such that

$$
\limsup _{t \rightarrow \infty}\left|x\left(t ; t_{0}, \phi\right)\right|=0 .
$$

But this is in contradiction with (36), and thus (34) must hold and this completes the proof.

(II) The proof of stochastic global asymptotic stability.

Give any $\varepsilon \in(0,1), \phi \in B C((-\infty, 0] ; \mathbb{R})$. Let $H$ be sufficiently large such that

$$
\inf _{|x|>H, t \geq t_{0}} V(x, t) \geq \frac{4 V\left(\phi\left(t_{0}\right), t_{0}\right)}{\varepsilon} .
$$

Then we can easily have

$$
\mathbb{E} V\left(x\left(t \wedge \tau^{H}\right), t \wedge \tau^{H}\right)<\mathbb{E} V\left(\phi\left(t_{0}\right), t_{0}\right) .
$$

From the assumption of $H$,

$$
\begin{aligned}
\mathbb{E} V\left(x\left(t \wedge \tau^{H}\right), t \wedge \tau^{H}\right) & \geq \mathbb{E}\left(I_{\left\{\tau^{H}<t\right\}} V\left(x\left(\tau^{H}\right), \tau^{H}\right)\right) \\
& \geq \frac{4 V\left(\phi\left(t_{0}\right), t_{0}\right)}{\varepsilon} \mathbb{P}\left(\tau^{H}<t\right) .
\end{aligned}
$$

So

$$
\mathbb{P}\left(\tau^{H}<t\right)<\frac{\varepsilon}{4} .
$$

Let $t \rightarrow \infty$. we could get

$$
\mathbb{P}\left(\tau^{H}<\infty\right)<\frac{\varepsilon}{4},
$$

namely,

$$
\mathbb{P}\left(\left|x\left(t ; t_{0}, \phi\right)\right| \leq H\right)>1-\frac{\varepsilon}{4} .
$$

Arguing as part (I), we obtain that

$$
\mathbb{P}\left(\lim _{t \rightarrow \infty} x\left(t ; t_{0}, \phi\right)=0\right)>1-\varepsilon .
$$

From the arbitrariness of $\varepsilon$, the trivial solution of (1) is stochastically globally asymptotically stable, which ends the proof.

In the last part of this section, we give two examples for better understanding the stability theorems above.

Example 7. Consider the following IDSVIDE:

$$
\begin{aligned}
\mathrm{d} x(t)= & {\left[\left(-2 e^{-t}-1\right) x(t)+\int_{-\infty}^{t} e^{s-2 t} x^{2}(s) \mathrm{d} s\right] \mathrm{d} t } \\
& +\left[e^{-t / 2} x(t)\right. \\
& \left.+\int_{-\infty}^{t} e^{s-\gamma t} x^{2}(s) \mathrm{d} s\right] \mathrm{d} W(t), \quad t \in[0,+\infty),
\end{aligned}
$$

Obviously, $|g(x)|=x^{2}$, so $J=1$. Let $h(s)=e^{s}, F(t)=e^{-t}$. Then we have $\int_{-\infty}^{0} h(s) \mathrm{d} s=1<\infty$ and

$$
\begin{gathered}
\int_{t}^{+\infty} F(u) \mathrm{d} u=e^{-t} \leq 1<\infty, \quad \lim _{t \rightarrow \infty} \int_{t}^{+\infty} F(u) \mathrm{d} u=0 \\
|C(t, s)|=e^{s-2 t}=e^{s-t} e^{-t}=F(t) h(s-t) .
\end{gathered}
$$

For any $\alpha \in(0,1)$, let $k=2$. Easily to get

$$
\begin{gathered}
B^{2}(t)=e^{-t}, \quad A(t)+k J \int_{t}^{+\infty}|C(u, t)| \mathrm{d} u=-e^{-t}-1, \\
m(t) D(t, s)=e^{-2 \gamma t+t+s}, \quad \frac{k-\alpha}{\sup _{|x| \leq \alpha}|g(x)|}|C(t, s)|>e^{s-2 t} .
\end{gathered}
$$

Therefore,

$$
\begin{aligned}
& B^{2}(t)<-\left(A(t)+k J \int_{t}^{+\infty}|C(u, t)| \mathrm{d} u\right), \\
& m(t) D(t, s)<\frac{k-\alpha}{\sup _{|x| \leq \alpha}|g(x)|}|C(t, s)| .
\end{aligned}
$$

Hence all the conditions of Theorem 3 have been verified, and it follows that the trivial solution of (1) is stochastically uniformly stable.

Example 8. Consider an IDSVIDE as follows:

$$
\begin{aligned}
& \mathrm{d} x(t)= {\left[\left(-\log ^{2}\left(x^{2}(t)+1\right)-2\right) x(t)\right.} \\
&\left.+\int_{-\infty}^{t} \frac{e^{-t}}{(t-s+2)^{2}} \sin \left(x^{2}(s)\right) \mathrm{d} s\right] \mathrm{d} t \\
&+\left[\log ^{2}\left(x^{2}(t)+1\right) x(t)\right. \\
&\left.+\int_{-\infty}^{t} e^{\beta s-\gamma t} \sin \left(x^{2}(s)\right) \mathrm{d} s\right] \mathrm{d} W(t), \\
& t \in[0,+\infty),
\end{aligned}
$$

where $\beta>4, \gamma>\beta+1$ are constants.

Obviously, $|g(x)|=\left|\sin x^{2}\right| \leq x^{2}$, so we can let $J=1$. Let $h(s)=1 /(1-s)^{2}, F(t)=e^{-t}$. Then we have $\int_{-\infty}^{0} h(s) \mathrm{d} s=1<$ $\infty$ and

$$
\begin{gathered}
\int_{t}^{+\infty} F(u) \mathrm{d} u=e^{-t} \leq 1<\infty, \quad \lim _{t \rightarrow \infty} \int_{t}^{+\infty} F(u) \mathrm{d} u=0 \\
|C(t, s)|=\frac{e^{-t}}{(t-s+2)^{2}} \leq \frac{e^{-t}}{(t-s+1)^{2}}=F(t) h(s-t) .
\end{gathered}
$$

where $\gamma>2$ is a constant. 
Additionally, for any $\alpha \in(0,1)$, let $k=2$ and $\delta=1 / 2$. Then it is not difficult to check that

$$
\begin{aligned}
B^{2}(t) & =\log ^{2}\left(x^{2}(t)+1\right)<\log ^{2}\left(x^{2}(t)+1\right)+\frac{1}{2} \\
& =-\left(A(t)+2 \int_{t}^{+\infty} \frac{1}{(u-t+2)^{2}} \mathrm{~d} u\right)-\frac{1}{2} \\
& \leq\left(A(t)+k J \int_{t}^{+\infty}|C(u, t)| \mathrm{d} u\right)-\delta .
\end{aligned}
$$

Consider function $f(u)=e^{\beta u}(u-2)^{2}-\beta, u<0$. Clearly, $f(u)$ is differentiable and

$$
\begin{gathered}
f^{\prime}(u)=e^{\beta u}(u-2)(\beta(u-2)+2)>0, \\
f(0)=4-\beta<0 .
\end{gathered}
$$

So $f(u)<0$, that is, $e^{\beta u} / \beta<1 /(u-2)^{2}$. Hence,

$$
\begin{aligned}
\sup _{|x| \leq \alpha}\left(\sin x^{2}\right) m(t) D(t, s) & \leq D(t, s) \int_{-\infty}^{t}|D(t, s)| \mathrm{d} s \\
& =\frac{1}{\beta} e^{(2 \beta-2 \gamma) t} e^{\beta s-\beta t} \\
& <\frac{e^{(2 \beta-2 \gamma) t}}{(s-t-2)^{2}}<|C(t, s)| .
\end{aligned}
$$

Hence, all of the conditions of Theorem 6 are satisfied and we can assert that the trivial solution of (1) is stochastically asymptotically stable and stochastically globally asymptotically stable.

\section{Boundedness of Solutions of IDSVIDEs}

In the section, we begin with three types of definitions about boundedness for solutions of (1).

Definition 9. (1) A solution $x(t)$ of (1) is stochastically bounded, if, for any $\varepsilon \in(0,1), \phi \in B C((-\infty, 0] ; \mathbb{R})$, there exists $R(\varepsilon, \phi)>0$, such that

$$
\mathbb{P}\left(\sup _{t \geq t_{0}}\left|x\left(t ; t_{0}, \phi\right)\right| \leq R\right)>1-\varepsilon .
$$

(2) The solutions of (1) are stochastically equibounded, if, for any $\varepsilon \in(0,1), \alpha>0$, and $\phi \in B C((-\infty, 0] ; \mathbb{R})$, there exists $R(\varepsilon, \alpha)>0$, such that

$$
\mathbb{P}\left(\sup _{t \geq t_{0}}\left|x\left(t ; t_{0}, \phi\right)\right| \leq R,\|\phi\|<\alpha\right)>1-\varepsilon .
$$

(3) Fix $\beta>0$; the solutions of (1) are stochastically ultimately bounded for bound $\beta$, if, for any $\varepsilon \in(0,1), \phi \in$ $B C((-\infty, 0] ; \mathbb{R})$, there exists a $T(\varepsilon, \phi) \geq t_{0}$, such that

$$
\mathbb{P}\left(\sup _{t \geq T(\varepsilon, \phi)}\left|x\left(t ; t_{0}, \phi\right)\right| \leq \beta\right)>1-\varepsilon .
$$

Now we apply the Lyapunov-Krasovskii method to give the sufficient conditions, under which the solutions of (1) are stochastically bounded, stochastically equibounded, and stochastically ultimately bounded, respectively.

Theorem 10. Suppose that (K1) and (K2) hold. If $f(t)=$ 0 , then the solutions to (1) are stochastically bounded and stochastically equibounded.

Proof. (I) Proof of stochastic boundedness.

Let $\varepsilon \in(0,1), \phi \in B C((-\infty, 0] ; \mathbb{R})$ be arbitrary. Choose $H$ sufficiently large such that $\inf _{|x|>H, t \geq t_{0}} V(x, t) \geq$ $V\left(\phi\left(t_{0}\right), t_{0}\right) / \varepsilon$. By the Itô formula, for any $t \geq t_{0}$, we can obtain that

$$
\begin{aligned}
& V\left(x\left(t \wedge \tau^{H}\right), t \wedge \tau^{H}\right) \\
& =V\left(\phi\left(t_{0}\right), t_{0}\right)+\int_{t_{0}}^{t \wedge \tau^{H}} \mathscr{L} V(x(s), s) \mathrm{d} s \\
& \quad+\int_{t_{0}}^{t \wedge \tau^{H}} V_{x}(x(s), s) \\
& \quad \times[B(s) x(s) \\
& \left.\quad+\int_{-\infty}^{s} D(s, r) g(x(r)) \mathrm{d} r+f_{1}(s)\right] \mathrm{d} W(s), \quad \text { a.s. }
\end{aligned}
$$

Taking expectation on both sides of (80), it follows by using (10) that

$$
\mathbb{E} V\left(x\left(t \wedge \tau^{H}\right), t \wedge \tau^{H}\right) \leq V\left(\phi\left(t_{0}\right), t_{0}\right) .
$$

From the assumption $H$, we derive that

$$
\begin{aligned}
\mathbb{E} V\left(x\left(t \wedge \tau^{H}\right), t \wedge \tau^{H}\right) & \geq \mathbb{E}\left(I_{\left\{\tau^{H}<t\right\}} V\left(x\left(\tau^{H}\right), \tau^{H}\right)\right) \\
& \geq \frac{V\left(\phi\left(t_{0}\right), t_{0}\right)}{\varepsilon} \mathbb{P}\left(\tau^{H}<t\right) .
\end{aligned}
$$

We therefore must have

$$
\mathbb{P}\left(\tau^{H}<t\right) \leq \varepsilon
$$

Letting $t \rightarrow \infty$, we can obtain that

$$
\mathbb{P}\left(\tau^{H}<\infty\right) \leq \varepsilon
$$

That is,

$$
\mathbb{P}\left(\sup _{t \geq t_{0}}|x(t)| \leq H\right)>1-\varepsilon
$$

The proof of stochastic boundedness is complete.

(II) Proof of stochastic equiboundedness.

Let $\varepsilon \in(0,1)$ and $\alpha>0$ be arbitrary. Give any $\phi \epsilon$ $B C((-\infty, 0] ; \mathbb{R})$, such that $\|\phi\| \leq \alpha$. Choose $R$ such that 
$R>\alpha^{2}(1+2 k J l a) / 2 \varepsilon$. By applying the Itô formula, for any $t \geq t_{0}$

$$
\begin{aligned}
& V\left(x\left(t \wedge \tau^{R}\right), t \wedge \tau^{R}\right) \\
& =V\left(\phi\left(t_{0}\right), t_{0}\right)+\int_{t_{0}}^{t \wedge \tau^{R}} \mathscr{L} V(x(s), s) \mathrm{d} s \\
& +\int_{t_{0}}^{t \wedge \tau^{R}} V_{x}(x(s), s) \\
& \quad \times\left[B(s) x(s)+\int_{-\infty}^{s} D(s, r) g(x(r)) \mathrm{d} r\right. \\
& \left.\quad+f_{1}(s)\right] \mathrm{d} W(s), \quad \text { a.s. }
\end{aligned}
$$

Taking the expectation for both sides of (86), we have

$$
\mathbb{E} V\left(x\left(t \wedge \tau^{R}\right), t \wedge \tau^{R}\right) \leq V\left(\phi\left(t_{0}\right), t_{0}\right) .
$$

In view of

$$
\begin{aligned}
& V\left(\phi\left(t_{0}\right), t_{0}\right)=\frac{\phi^{2}\left(t_{0}\right)}{2}+k \int_{-\infty}^{t_{0}} \int_{t_{0}}^{+\infty}|C(u, s)||g(x(s))| \mathrm{d} u \mathrm{~d} s \\
& \leq\left(\frac{1}{2}+k J l a\right)\|\phi\|^{2}, \\
& \mathbb{E} V\left(x\left(t \wedge \tau^{R}\right), t \wedge \tau^{R}\right) \geq \mathbb{E}\left(I_{\left\{\tau^{R}<t\right\}} V\left(x\left(\tau^{R}\right), \tau^{R}\right)\right) \\
& \geq R \mathbb{P}\left(\tau^{R}<t\right) .
\end{aligned}
$$

Consequently,

$$
\mathbb{P}\left(\tau^{R}<t\right) \leq \frac{(1+2 k J l a)\|\phi\|^{2}}{2 R} \leq \frac{\alpha^{2}(1+2 k J l a)}{2 R}<\varepsilon .
$$

Letting $t \rightarrow \infty$, it yields that

$$
\mathbb{P}\left(\tau^{R}<\infty\right) \leq \varepsilon
$$

that is,

$$
\mathbb{P}\left(\sup _{t \geq t_{0}}|x(t)| \leq R,\|\phi\|<\alpha\right)>1-\varepsilon .
$$

This completes the proof.

Remark 11. Notice that, when conditions (K1) and (K2) hold, the solutions of stochastic system (1) are stable and bounded. That is, environmental noise (in the sense of Itô) cannot disturb stability and boundedness of solutions for some systems if noisy intensity satisfies (K2). Hence, we can construct some anti-interference systems in practice.

Theorem 12. Suppose that (K1) and (K3) hold. If $f(t)=0$, then the solutions to (1) are stochastically ultimately bounded.
Proof. Give $\beta>0$. For any $\varepsilon \in(0,1), \phi \in B C((-\infty, 0] ; \mathbb{R})$. From Theorem 10, there exists $H>0$, such that

$$
\mathbb{P}\left(|x(t)| \leq H, t \geq t_{0}\right) \geq 1-\frac{\varepsilon}{4} .
$$

Choose $\alpha<\left|\phi\left(t_{0}\right)\right| \wedge \beta$, and define four stopping times as follows:

$$
\begin{aligned}
\tau_{\alpha} & =\inf \left\{t \geq t_{0}:|x(t)| \leq \alpha\right\}, \\
\tau^{H} & =\inf \left\{t \geq t_{0}:|x(t)| \geq H\right\}, \\
\tau^{\beta} & =\inf \{t>\sigma:|x(t)| \geq \beta\}, \\
\sigma & = \begin{cases}\tau_{\alpha}, & \tau_{\alpha}<\tau^{H} \wedge \theta_{\alpha} ; \\
\infty, & \text { otherwise. }\end{cases}
\end{aligned}
$$

From here, we can show in the same way as in the proof of Theorem 6 that we could choose sufficiently small $\alpha$, such that $2 \alpha^{2} / H^{2}<\varepsilon$ and

$$
\begin{aligned}
\frac{H^{2}}{2} \mathbb{P}\left(\tau^{\beta}<t\right) & \leq \mathbb{E}\left(I_{\left\{\tau_{\alpha}<\tau^{H} \wedge \theta_{\alpha}\right\}} V\left(x\left(\tau^{\beta} \wedge t\right), \tau^{\beta} \wedge t\right)\right) \\
& \leq \mathbb{E}\left(I_{\left\{\tau_{\alpha}<\tau^{H} \wedge \theta_{\alpha}\right\}} V\left(x\left(\tau_{\alpha}\right), \tau_{\alpha}\right)\right) \leq \alpha^{2} .
\end{aligned}
$$

Then it follows that

$$
\mathbb{P}\left(\tau^{\beta}<t\right)<\frac{2 \alpha^{2}}{H^{2}}<\varepsilon .
$$

After letting $t \rightarrow \infty$, we have

$$
\mathbb{P}\left(\tau^{\beta}<\infty\right) \leq \varepsilon .
$$

Let $T(\varepsilon, \phi)=\tau_{\alpha}$. Then the above inequality is equivalent to

$$
\mathbb{P}\left(\sup _{t \geq T}|x(t)| \leq \beta\right)>1-\varepsilon .
$$

The proof is complete.

Remark 13. Obviously, we can verify that the solutions of IDSVIDE (68) are stochastically bounded and stochastically equibounded. And the solutions of IDSVIDE (72) are stochastically ultimately bounded.

\section{Conclusions}

Throughout this paper, by combining the Lyapunov-Krasovskii method, we have obtained various kinds of sufficient stability and boundedness criteria, where the ranges of noisy intensity that stable and bounded systems can tolerate without losing the properties of stability and boundedness are presented, respectively. These sufficient conditions are very necessary for us to verify stability and boundedness of stochastic Volterra integrodifferential equations with infinite delays. Moreover, the conditions obtained in this paper can also help us to construct some antidisturbance systems in the applications. In addition, two examples have been given to illustrate our theoretical results. 


\section{Acknowledgments}

The authors really appreciate the reviewers' valuable comments and the reviewers' helpful suggestions to improve the paper. This work was supported by the NNSF of China (nos. 11171081, 11171056, and 11271065), the NNSF of Shandong Province (no. ZR2010AQ021), the Key Project of Science and Technology of Weihai (no. 2011dxgj06), and the Natural Scientific Research Innovation Foundation in Harbin Institute of Technology (no. HIT.NSRIF.2011104).

\section{References}

[1] F. Wei and K. Wang, "The existence and uniqueness of the solution for stochastic functional differential equations with infinite delay," Journal of Mathematical Analysis and Applications, vol. 331, no. 1, pp. 516-531, 2007.

[2] Y. Ren, S. Lu, and N. Xia, "Remarks on the existence and uniqueness of the solutions to stochastic functional differential equations with infinite delay," Journal of Computational and Applied Mathematics, vol. 220, no. 1-2, pp. 364-372, 2008.

[3] Y. Ren and N. Xia, "Existence, uniqueness and stability of the solutions to neutral stochastic functional differential equations with infinite delay," Applied Mathematics and Computation, vol. 210, no. 1, pp. 72-79, 2009.

[4] Y. Ren and L. Chen, "A note on the neutral stochastic functional differential equation with infinite delay and Poisson jumps in an abstract space," Journal of Mathematical Physics, vol. 50, no. 8, Article ID 082704, 8 pages, 2009.

[5] H. Bao and J. Cao, "Existence and uniqueness of solutions to neutral stochastic functional differential equations with infinite delay," Applied Mathematics and Computation, vol. 215, no. 5, pp. 1732-1743, 2009.

[6] F. Wu and S. Hu, "Khasminskii-type theorems for stochastic functional differential equations with infinite delay," Statistics \& Probability Letters, vol. 81, no. 11, pp. 1690-1694, 2011.

[7] B. de Andrade, C. Cuevas, and E. Henríquez, "Asymptotic periodicity and almost automorphy for a class of Volterra integro-differential equations," Mathematical Methods in the Applied Sciences, vol. 35, no. 7, pp. 795-811, 2012.

[8] Q. C. Huang and K. Wang, "Space $C_{h}$, boundedness and periodic solutions of FDE with infinite delay," Scientia Sinica. Series A, vol. 30, no. 8, pp. 807-818, 1987.

[9] X. Mao, "Stability of stochastic integro-differential equations," Stochastic Analysis and Applications, vol. 18, no. 6, pp. 1005-1017, 2000.

[10] B. Zhang, "Uniform asymptotic stability in functionaldifferential equations with infinite delay," in Comparison Methods and Stability Theory (Waterloo, ON, 1993), vol. 162 of Lecture Notes in Pure and Applied Mathematics, pp. 349-362, Dekker, New York, NY, USA, 1994.

[11] W. Li, H. Su, and K. Wang, "Global stability analysis for stochastic coupled systems on networks," Automatica, vol. 47, no. 1, pp. 215-220, 2011.

[12] M. Y. Li and Z. Shuai, "Global-stability problem for coupled systems of differential equations on networks," Journal of Differential Equations, vol. 248, no. 1, pp. 1-20, 2010.

[13] W. Yu, J. Cao, and J. Wang, "An LMI approach to global asymptotic stability of the delayed Cohen-Grossberg neural network via nonsmooth analysis," Neural Networks, vol. 20, no. 7, pp. 810-818, 2007.
[14] S. Zhou, Z. Wang, and D. Feng, "Stochastic functional differential equations with infinite delay," Journal of Mathematical Analysis and Applications, vol. 357, no. 2, pp. 416-426, 2009.

[15] Y. Hu, F. Wu, and C. Huang, "Robustness of exponential stability of a class of stochastic functional differential equations with infinite delay," Automatica, vol. 45, no. 11, pp. 2577-2584, 2009.

[16] F. Wu, S. Hu, and C. Huang, "Robustness of general decay stability of nonlinear neutral stochastic functional differential equations with infinite delay," Systems \& Control Letters, vol. 59, no. 3-4, pp. 195-202, 2010.

[17] Z. Yang, E. Zhu, Y. Xu, and Y. Tan, "Razumikhin-type theorems on exponential stability of stochastic functional differential equations with infinite delay," Acta Applicandae Mathematicae, vol. 111, no. 2, pp. 219-231, 2010.

[18] X. Li and X. Fu, "Stability analysis of stochastic functional differential equations with infinite delay and its application to recurrent neural networks," Journal of Computational and Applied Mathematics, vol. 234, no. 2, pp. 407-417, 2010.

[19] $\mathrm{F}$. $\mathrm{Wu}$ and $\mathrm{S}$. $\mathrm{Hu}$, "Attraction, stability and robustness for stochastic functional differential equations with infinite delay," Automatica, vol. 47, no. 10, pp. 2224-2232, 2011.

[20] H. Su, W. Li, K. Wang, and X. Ding, "Stability analysis for stochastic neural network with infinite delay," Neurocomputing, vol. 74, no. 10, pp. 1535-1540, 2011.

[21] G. Pavlović and S. Janković, "Razumikhin-type theorems on general decay stability of stochastic functional differential equations with infinite delay," Journal of Computational and Applied Mathematics, vol. 236, no. 7, pp. 1679-1690, 2012.

[22] C. Zhang and S. Vandewalle, "Stability analysis of Volterra delay-integro-differential equations and their backward differentiation time discretization," Journal of Computational and Applied Mathematics, vol. 164, no. 1, pp. 797-814, 2004.

[23] P. Eloe, M. Islam, and B. Zhang, "Uniform asymptotic stability in linear Volterra integrodifferential equations with application to delay systems," Dynamic Systems and Applications, vol. 9, no. 3, pp. 331-344, 2000.

[24] T. A. Burton, Volterra Integral and Differential Equations, vol. 202 of Mathematics in Science and Engineering, Elsevier B. V., Amsterdam, The Netherlands, 2nd edition, 2005.

[25] R. Qi, C.-J. Zhang, and Y.-J. Zhang, "Dissipativity of multistep Runge-Kutta methods for nonlinear Volterra delay-integrodifferential equations," Acta Mathematicae Applicatae Sinica. English Series, vol. 28, no. 2, pp. 225-236, 2012.

[26] X. Mao and M. Riedle, "Mean square stability of stochastic Volterra integro-differential equations," Systems \& Control Letters, vol. 55, no. 6, pp. 459-465, 2006.

[27] J. A. D. Appleby and M. Riedle, "Almost sure asymptotic stability of stochastic Volterra integro-differential equations with fading perturbations," Stochastic Analysis and Applications, vol. 24, no. 4, pp. 813-826, 2006.

[28] X. Mao, Stochastic Differential Equations and Their Applications, Horwood Publishing Series in Mathematics \& Applications, Horwood Publishing, Chichester, UK, 1997.

[29] J. K. Hale and J. Kato, "Phase space for retarded equations with infinite delay," Funkcialaj Ekvacioj. Serio Internacia, vol. 21, no. 1, pp. 11-41, 1978.

[30] Y. Hino, S. Murakami, and T. Naito, Functional-Differential Equations with Infinite Delay, vol. 1473 of Lecture Notes in Mathematics, Springer, Berlin, Germany, 1991. 


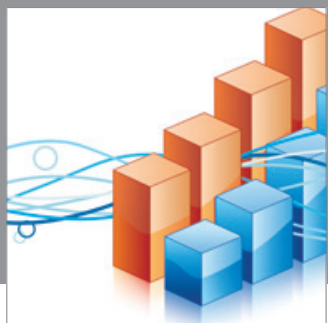

Advances in

Operations Research

mansans

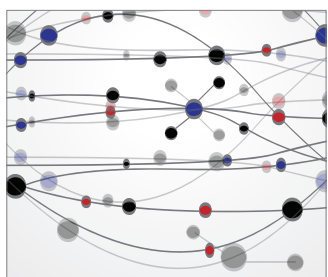

The Scientific World Journal
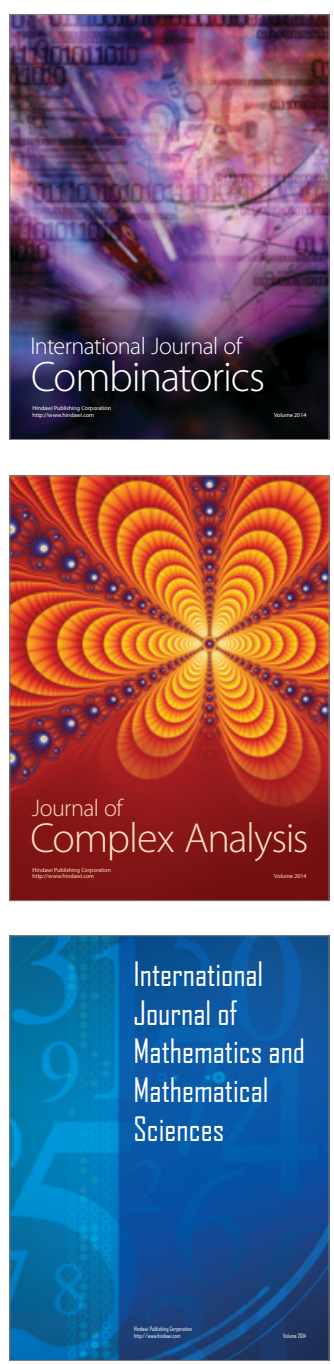
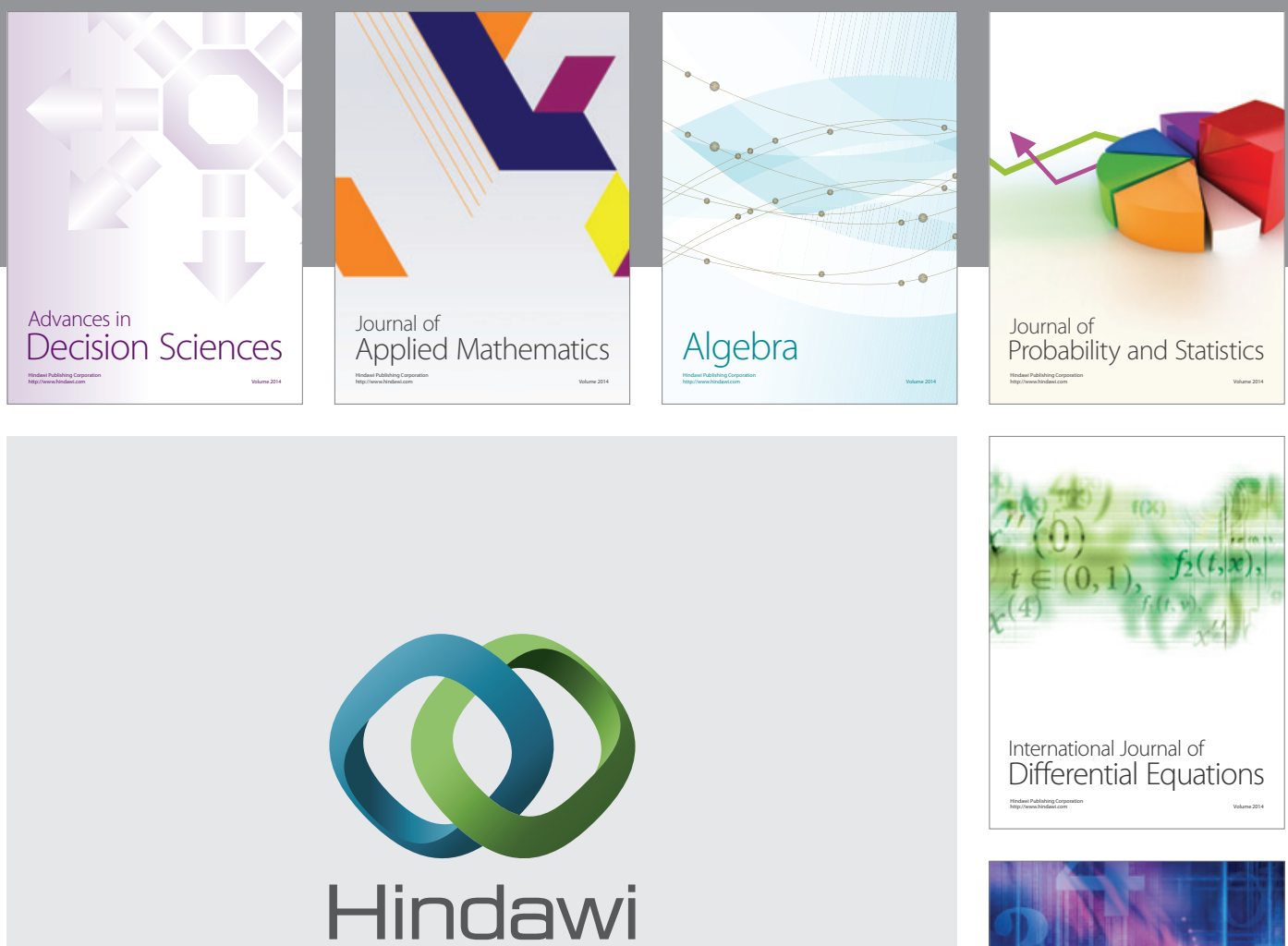

Submit your manuscripts at http://www.hindawi.com
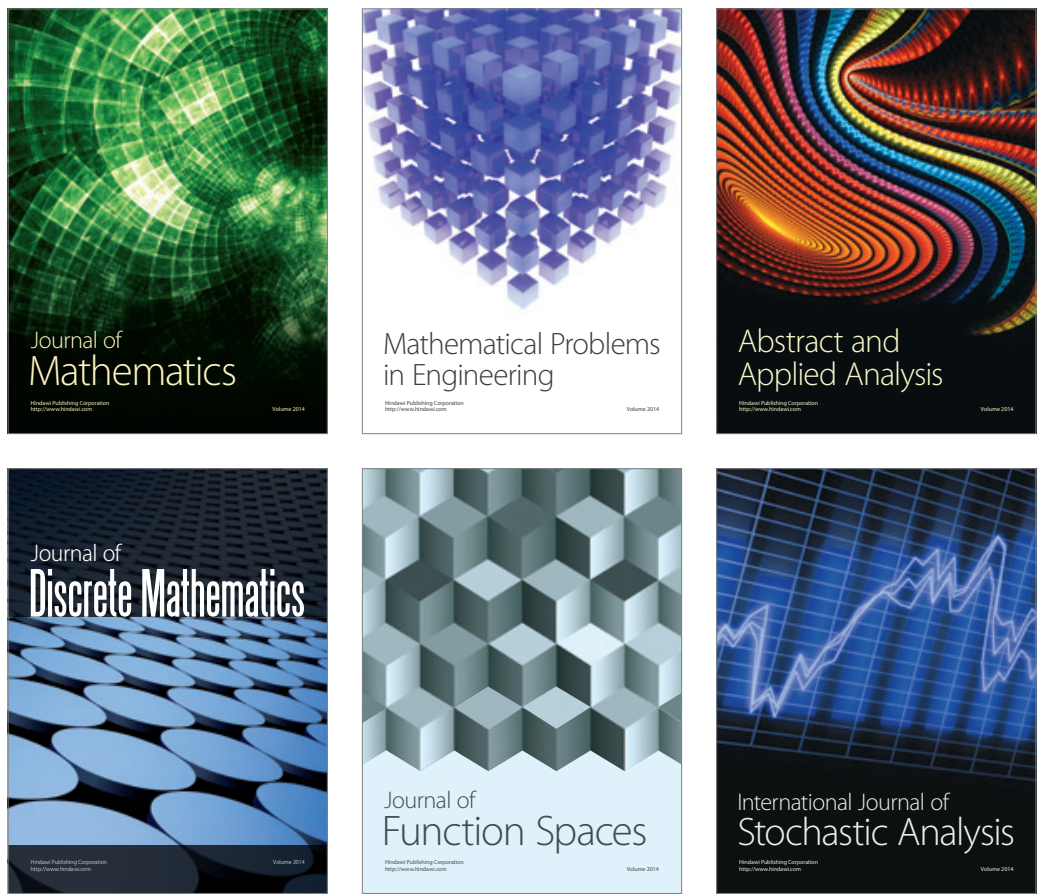

Journal of

Function Spaces

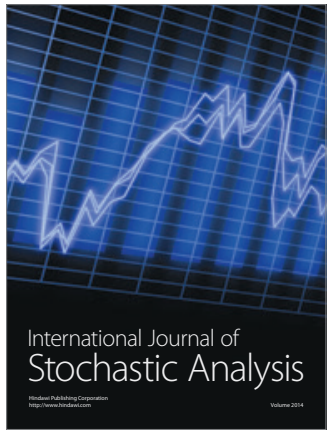

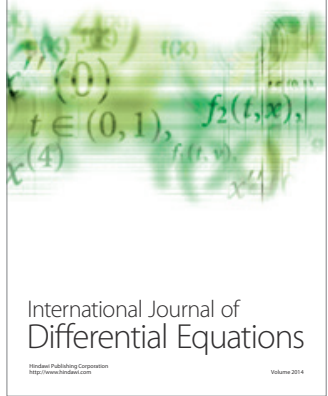
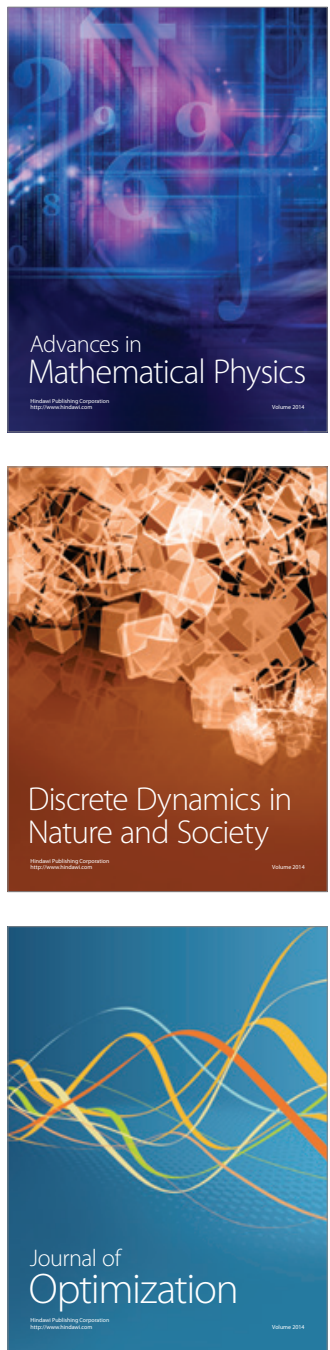